\title{
Chlorhexidine varnish has caries-reducing potential
}

\author{
Is a 6-monthly application of $40 \%$ chlorhexidine varnish effective in reducing \\ dental caries in primary molars?
}

\begin{abstract}
Du MQ, Tai BJ, Jiang H, Lo EC, Fan MW, Bian Z. A two-year randomized clinical trial of chlorhexidine varnish on dental caries in Chinese preschool children. J Dent Res 2006;85:557-559
\end{abstract}

Design A double-blind, randomised, placebo-controlled clinical trial was conducted.

Intervention Children in the test group received 6-monthly applications of a $40 \%$ chlorhexidine varnish whereas children in the control group received a placebo varnish, over the course of 2 years. Clinical examination was undertaken at baseline and after 24 months by two calibrated dentists who did not know to which groups children were assigned.

Outcome measure Decayed, missing, or primary filled molar surfaces (dmfs-molar) were monitored.

Results At baseline, there was no significant difference between the mean dmfs-molar scores of the two groups (2.8 vs 2.6 ; $\mathrm{P}>0.05-$ ). After 2 years, 44 children (13\%) were lost to follow-up, because some $(n=31)$ had moved to other kindergartens, and some $(n=13)$ objected to the taste of the varnish and refused to be examined. After 2 years, 290 children remained in the study, of whom 155 were in the test group and 135 were in the placebo group. The mean caries increment of the primary molars was $1.0 \mathrm{dmfs}-$ molar in the test-group children and 1.6 dmfs-molar in the placebo group. The difference of 0.6 tooth surface equated to a $37.3 \%$ reduction in caries increment (number-needed-totreat of 3), and was statistically significant (P 0.036; 95\% confidence interval, 0.04-1.16). No side-effects (such as soft-tissue lesions and staining of teeth) were found at the 24-month examination.

Conclusions Six-monthly applications of chlorhexidine varnish were effective in reducing the incidence of dental caries in primary molars.

\section{Commentary}

The essential role of oral micro-organisms, in particular Streptococcus mutans, in the aetiology of dental caries is well-recognised. Since it is an effective antimicrobial agent, it seems reasonable that applying chlorhexidine as a varnish may be useful in caries reduction: indeed, over the last decade, a number of trials have taken place with the objective of determining the caries-preventive properties of chlorhexidine. The results of these studies, however, have been somewhat equivocal, some demonstrating a positive effect whereas others suggested little or no benefit.

This study by Du and colleagues describes a clinical trial of a $40 \%$ chlorhexidine varnish versus a placebo varnish not containing chlorhexidine. The research involved 334 children aged 4-5 years attending kindergartens in Hubei province in China. The study participants were randomised to the test or control treatment on a class basis (cluster allocation). The authors were careful to point out the assignment of children to classes in the study schools was not done on any special basis such as demographic or socio-economic background. Importantly, at baseline the mean dmfs-molar scores were 2.8 and $2.6 \mathrm{dmfs}$ in the test and placebo groups respectively and these differences were not statistically significant. The authors were careful to account for follow-up and to take steps to ensure that the study contained sufficient children to demonstrate a difference, if one existed (ie, the trial was of adequate statistical power).

The study showed a caries-preventive effect of chlorhexidine in this population. The finding of a $37.3 \%$ reduction in caries prevalence is impressive, at face value, as is the number-needed-to-treat value of 3 . Nevertheless, despite this is being a very well-conducted and -described study, there are a number of important factors to bear in mind in when considering the applicability of these results to other populations.

First is that caries diagnosis was carried out according to World Health Organization criteria (into dentine) and radiographs were not used, so the true caries experience may have been underscored. It also relates only to the deciduous dentition.

Most importantly, perhaps, when extrapolating the value of chlorhexidine varnish into Western European or North American populations, exposure to fluoride in this study population was probably low. The authors indicate that fluoride concentration in the local water supply was low $(0.1-0.3 \mathrm{ppm})$, and that no organised healthcare programs were available in the study site. No data are given on home fluoride use. Assuming this was low, then an important question remains: to what extent would such a caries reduction have been present if the children were exposed to an optimum fluoride dosage, including daily brushing with a fluoridated dentifrice?

Thus, although this study has shown that chlorhexidine has caries-reducing potential, whether this benefit would persist in Western communities exposed to greater use of fluoride, and whether in such circumstances its use in a public health programme would be cost-effective or cost-efficient, requires further investigation.

\section{Ivor G Chestnutt}

Department of Dental Health and Biological Sciences, Cardiff

University Dental School, Cardiff, Wales, UK

Evidence-Based Dentistry (2006) 7, 81-82. doi:10.1038/sj.ebd.6400443 